\title{
Peripheral Arteriovenous Malformations with a Dominant Outflow Vein: Results of Ethanol Embolization
}

\author{
Sung Ki Cho, MD ${ }^{1}$ \\ Young Soo Do, MD' \\ Dong Ik Kim, MD² \\ Young Wook Kim, MD² \\ Sung Wook Shin, MD' \\ Kwang Bo Park, MD' \\ Justin Sang Ko, MD \\ Ae Ryoung Lee, $\mathrm{MD}^{3}$ \\ Sung Wook Choo, MD' \\ In Wook Choo, MD ${ }^{1}$
}

\section{Index terms:}

Arteriovenous malformations, extremities

Arteriovenous malformations, pelvic

Arteriovenous malformations, therapeutic embolization

DOI:10.3348/kjr.2008.9.3.258

Korean J Radiol 2008; $9: 258-267$

Received October 19, 2007; accepted

after revision February 9, 2007.

Departments of 'Radiology and Center for Imaging Science, ${ }^{2}$ Surgery,

${ }^{3}$ Anesthesiology and Pain Medicine,

Samsung Medical Center, Sungkyunkwan University School of Medicine, Seoul 135710 , Korea

Address reprint requests to:

Young Soo Do, MD, Department of

Radiology and Center for Imaging

Science, Samsung Medical Center,

Sungkyunkwan University School of

Medicine, 50 Irwon-dong, Gangnam-gu,

Seoul 135-710, Korea.

Tel. (822) 3410-2518

Fax. (822) 3410-2559

e-mail: ysdo@skku.edu
Objective: To assess retrospectively the treatment results of ethanol embolization of peripheral arteriovenous malformations (AVMs) with a dominant outflow vein (DOV).

Materials and Methods: Nineteen patients who had peripheral AVMs with a DOV were enrolled in this study (mean age, 29.7 years; range, $15-42$ years). Fifty-one ethanol embolizations (mean, 2.7; range, 1 -8) were performed by direct puncture $(n=29)$, the transarterial approach $(n=13)$, the transvenous approach $(n=5)$, or a combination of methods $(n=4)$ under general anesthesia. Coil and/or core-removed guide wire embolization of the DOV or another flow occlusion technique (i.e., use of an external pneumatic pressure cuff) to achieve vascular stasis were required in all patients during ethanol embolization. Clinical follow-up (mean, 22.2 months; range, 1-53 months) was performed for all patients, and imaging follow-up (mean, 22.1 months; range, 2 -53 months) from the last treatment session was performed for 14 patients. The therapeutic outcome (cure, improvement, no change, or aggravation) was assessed according to the clinical response and the degree of devascularization at angiography.

Results: Ethanol embolization was considered as an effective procedure in all patients. Thirteen (68\%) of 19 patients were cured and six displayed improvement. Three of six patients with improvement needed further treatment sessions for residual AVMs. Four patients (21\%) experienced a total of eight complications. Five complications (three events of a distal embolism and one event each of a urinary bladder necrosis and a brain infarct related to the accidental cannulation of the common carotid artery during insertion of the Swan-Ganz catheter) were major and three complications (skin necrosis) were minor.

Conclusion: Peripheral AVMs with a DOV can be effectively treated with a high cure rate by the use of ethanol embolization alone or in conjunction with the use of coil and/or core-removed guide wire embolization.

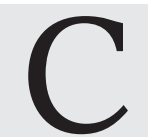

ongenital arteriovenous malformations (AVMs) are a major challenge to treat in medical practice, and are often associated with serious symptoms such as heart failure, neuropathy, pain, and bleeding (1-3). Small and superficial AVMs can be cured with surgical resection; however, most AVMs are inoperable as they are large and diffuse in nature, and they involve important normal adjacent structures. With improvement of catheter technology, superselective techniques, and the use of liquid embolic agents, embolotherapy has emerged as the primary mode of treatment for the management of peripheral AVMs (3-11). Nonetheless, the heterogeneity and rarity of peripheral AVMs are obstacles that make it difficult for interventional radiologists to treat peripheral AVMs effectively.

The target of embolotherapy is the nidus of AVMs, which is an aggregation of arteri- 
ovenous shunts. Recently, to aid in the therapeutic decision making for performing embolotherapy, Cho et al. (12) have proposed four prototypes of peripheral AVMs based on the angiographic morphology of the nidus - these are arterio-venous fistulae, arteriolo-venous fistulae, arteriolovenulous fistulae with a non-dilated fistula, and arteriolovenulous fistulae with a dilated fistula. Simultaneously, these investigators reported that the treatment result of ethanol embolization for the arteriolo-venous fistulae type was better than the treatment result of ethanol embolization for the other types of fistulae. The AVM type of arteriolo-venous fistula has been referred to as an AVM with a dominant outflow vein (DOV) as the dilated venous component of the nidus appears as the part of a large outflow vein on angiography (13). A few other studies have reported excellent results with the use of embolotherapy for treating peripheral AVMs with a DOV $(9,13)$. Thus, we believe that special attention needs to be applied to AVMs with a DOV and it is necessary to detail the procedural aspects of embolotherapy for this subset of peripheral AVMs.

The purpose of this study was to assess retrospectively the therapeutic results of ethanol embolization of peripheral AVMs with a DOV and to describe the procedural details of ethanol embolization for this type of AVM.

\section{MATERIALS AND METHODS}

\section{Patients}

Our institutional review board approved this retrospective review of patient medical and imaging records and waived the requirement of informed consent for the study. Written consent for undergoing a procedure was obtained from the all patients after a discussion about the advantages and risks associated with ethanol embolization.

From November 1996 to November 2006, 111 consecutive patients with inoperable peripheral AVMs underwent staged ethanol embolizations. As in previous studies $(9,12$, 14), AVMs with a DOV were defined when multiple arteriolar components of the nidus shunted into a dilated venous component of the nidus that was identified as the part of a large outflow vein on angiography (Fig. 1). According to the angiographic classification proposed by Cho et al. (12), all the angiographic studies of the 111 patients were retrospectively reviewed by consensus of two radiologists. Nineteen patients were considered to have AVMs with a DOV.

The 19 patients consisted of six men and 13 women (mean age: 29.7 years, age range: $15-42$ years). Ethanol embolization was the primary treatment modality for 15 patients. The remaining four patients had undergone previous unsuccessful surgery and/or feeding artery embolization with the use of microcoils. The 19 patients

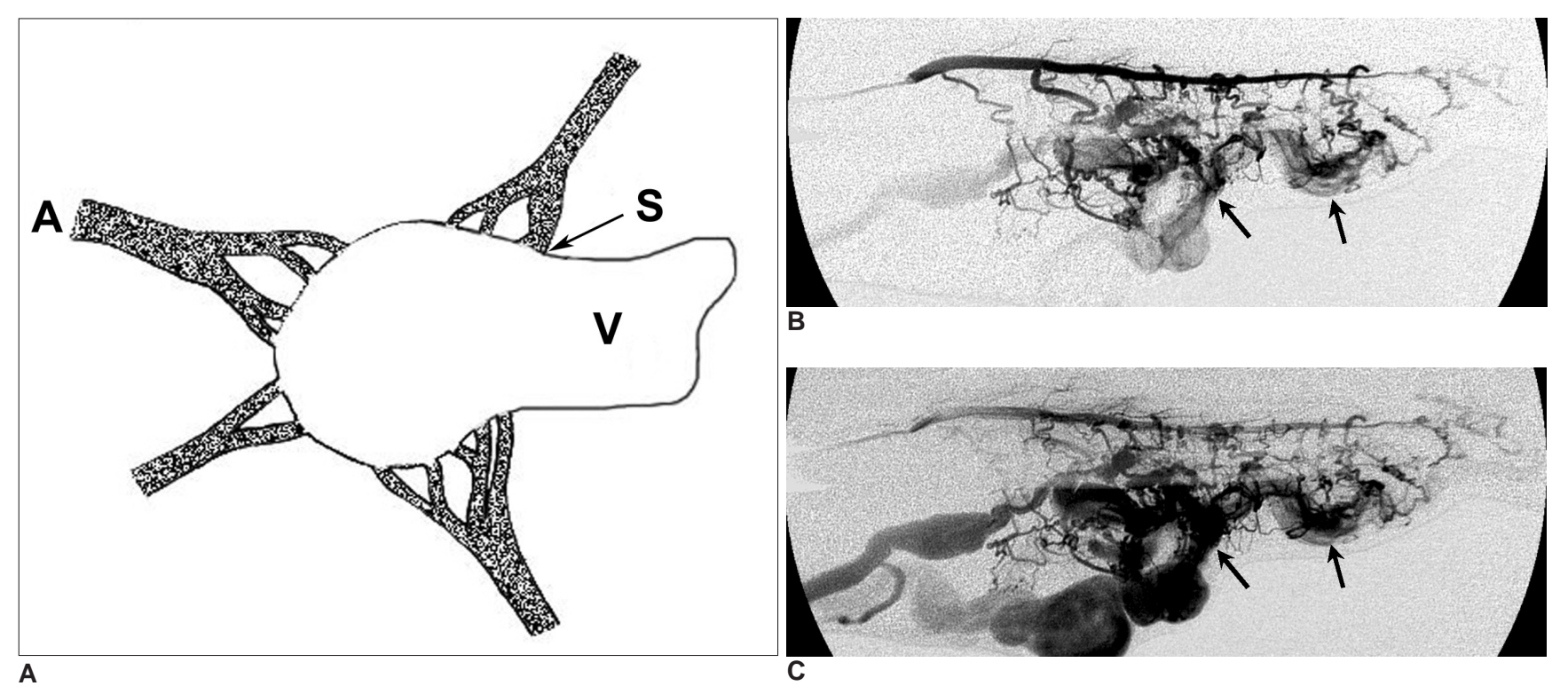

Fig. 1. Diagram and angiographic example of peripheral arteriovenous malformations with dominant outflow vein.

A. Peripheral arteriovenous malformations with dominant outflow vein have arteriovenous shunts (S) between multiple arterial components $(A)$ and dilated venous component $(V)$ of nidus. Dilated venous component of nidus appears as initial part of large outflow vein on angiography.

B, C. Arterial (B) and venous (C) phases of selective posteroanterior angiogram of arteriovenous malformation with dominant outflow vein in left forearm show multiple arterial feeders that shunt into wall of large dilated outflow vein (arrows). 


\section{Cho et al.}

displayed various presenting symptoms and signs (Table 1). Pain (68\%, 13 of 19 patients) was the most common symptom. The location of the AVMs was the pelvic wall (n $=4)$, the calf $(n=4)$, the chest wall $(n=3)$, the hand $(n=$ $2)$, the uterus $(n=1)$, the shoulder $(n=1)$, the foot $(n=1)$, the forearm and hand $(n=1)$, the back muscle $(n=1)$, and the retroperitoneum $(n=1)$. The presence of an AVM was confirmed by a combination of clinical examination and the use of imaging studies including magnetic resonance imaging (MRI), computed tomography (CT) with 3dimensional reconstruction, color Doppler flow imaging, technetium 99m-labeled red blood cell scintigraphy, and/or diagnostic angiography.

\section{Embolization Procedures and Clinical Follow-Up}

All ethanol embolizations were performed under general anesthesia by three interventional radiologists. A SwanGanz catheter (Baxter Healthcare, Irvine, CA) to monitor pulmonary artery pressure was inserted at the first session of ethanol embolization in all patients. During subsequent sessions of ethanol embolization, the Swan-Ganz catheter was inserted only when the predicted single injection dose of absolute ethanol was greater than $3 \mathrm{~mL}$ and the total amount exceeded $0.25 \mathrm{~mL}$ per kilogram of body weight (15). For every embolization session, baseline selective and superselective angiographic studies were obtained to determine precisely the flow characteristics of the AVMs.

Transarterial or transvenous catheterization using a coaxial catheter, and/or percutaneous direct puncture were needed to reach the nidus being embolized. Initially, the transarterial approach was preferentially used to embolize AVMs when possible, as described in previous studies (8, 10). However, in this subset of peripheral AVMs where the nidus consisted of multiple arteriolar components and a dilated venous component, we found that superselective positioning of a catheter to the multiple tortuous arteriolar components of the nidus required much more time than approaching the dilated venous component of the nidus via either a direct puncture or via the transvenous approach. Furthermore, transarterial embolization was often abandoned because either severe tortuosity of the arteriolar components in the nidus or normal arteriolar branches arising in very close proximity to the nidus interfered with accessing to a location that allowed safe embolization.

Thereafter, either the direct puncture or transvenous approach was primarily used for embolizing this type of AVM. When embolization via percutaneous direct puncture was indicated, the area of percutaneous puncture was prepared and draped. Subsequently, an 18- or 21guage needle (Disposable Cook Modified Needle, Cook, Bloomington, IN) was advanced under fluoroscopic guidance.

As all the lesions had a large outflow vein, a few flow occlusion techniques were applied to achieve vascular stasis (9). An intravascular occlusion balloon catheter (Standard Occlusion Balloon Catheter, Boston Scientific, Natick, MA) was used for lesions located in the trunk and pelvic area, and external pneumatic pressure cuffs were used for lesions located in the extremities. However, the use of coil embolization (Nester coil, Cook, Bloomington, IN) of the large outflow vein via direct puncture or transvenous catheterization prior to ethanol injection was preferred as it allows for a reduction of the amount of ethanol used and allows for stabilization of the thrombosis that forms in the large venous component $(10,13)$. Coil embolization was not performed for those superficial lesions that were very close to the skin. For the AVMs with a larger outflow vein where coil embolization was not adequate and where the application of an external pneumatic pressure cuff was not possible, core-removed guide wire embolization of the outflow vein via direct puncture or transvenous catheterization was performed prior to ethanol injection. The core-removed guide wire used was a 0.035 -inch conventional metal guide wire with a length of $150 \mathrm{~cm}$ (M.I.Tech, Seoul, Korea) from which the inner metallic core was removed.

To determine the volumes of ethanol and injection rates, test injections of contrast medium were made under fluoroscopic monitoring. The amount of ethanol used was based on the amount of contrast medium required to fill the AVM nidus without opacifying the normal vessels. Absolute ethanol (99.9\%, J.T. Baker, Selangor, Malaysia) was used in most cases, and $80 \%$ ethanol diluted with nonionic contrast medium (Xenetix 300, Guerbet, Cedex, France) was used for the superficial lesions that were located very close to the skin. The total amount of ethanol used in a single session was restricted to $<1 \mathrm{~mL} / \mathrm{kg}$ of body weight $(3,15)$. Arteriography was performed 5 to 10 minutes after the ethanol injection to determine whether the AVMs were completely embolized. Meticulous repetition of these techniques was required for complete embolization of at least one compartment of the AVMs.

When the mean pulmonary artery pressure rose above $25 \mathrm{mmHg}$, nitroglycerine (Nitrolingual, Pohl-Boskamp, Hohenlockstedt, Germany) was administered as a bolus injection $(50-100 \mathrm{gg})$ and was then infused continuously at $0.3-3.0 \mu \mathrm{g} / \mathrm{kg} / \mathrm{min}$ through the Swan-Ganz catheter until the mean pulmonary artery pressure reached normal levels. If the pulmonary artery pressure remained elevated at the end of the session or if a large amount of absolute ethanol had been injected, the patient was kept in the intensive care unit for close pulmonary artery pressure 
Ethanol Embolization in Peripheral AVM with Dominant Outflow Vein

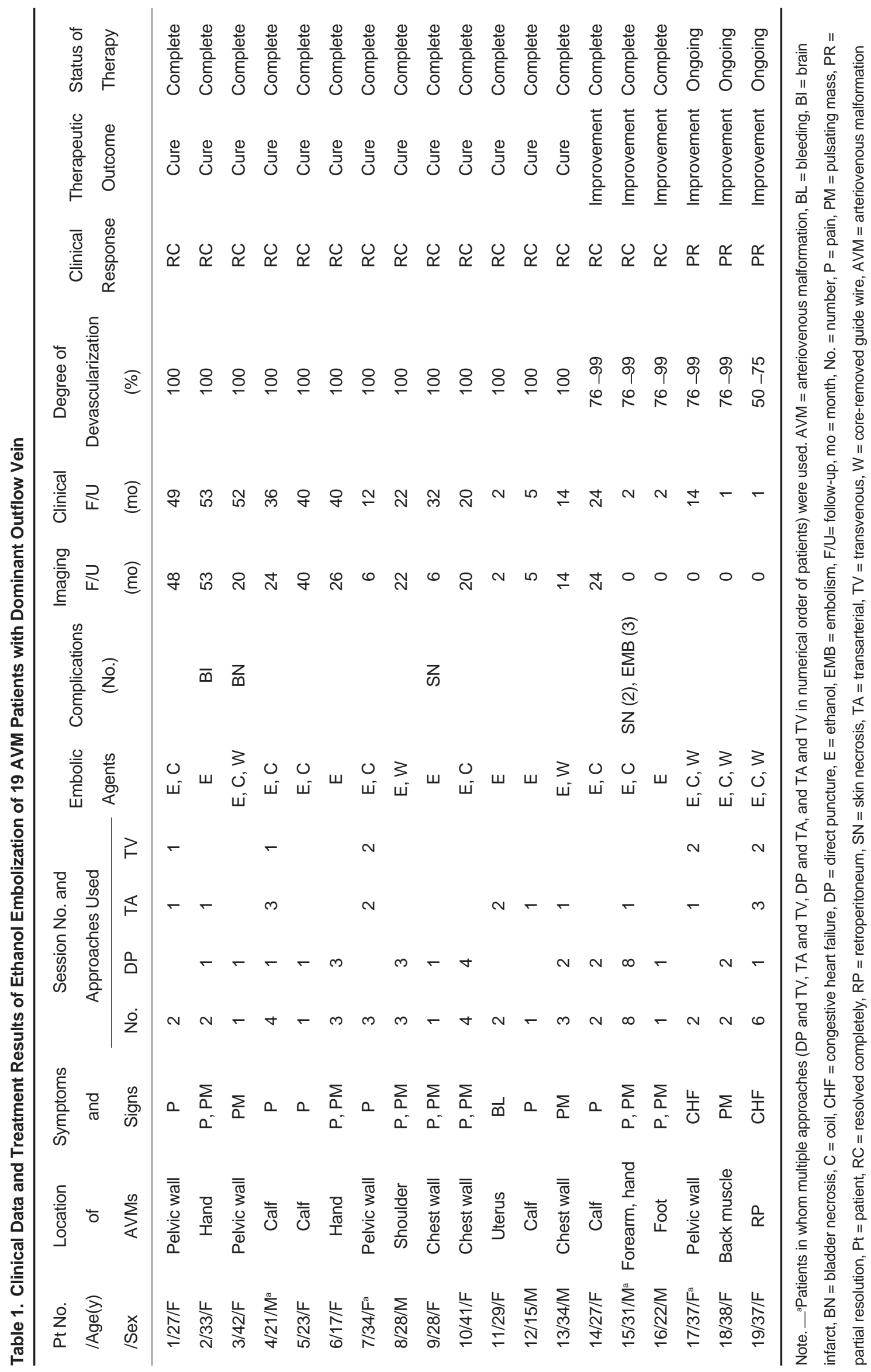




\section{Cho et al.}

monitoring and continuous administration of nitroglycerine. To control swelling, systemic dexamethasone ( 0.1 $\mathrm{mg} / \mathrm{kg}$ ) (Dexamethansone, Yuhan, Seoul, Korea) was administered immediately before the procedure and every eight hours while the patients were in the hospital. After the patients were discharged, prednisolone (Solondo, Yuhan, Seoul, Korea) was administered at $1 \mathrm{mg} / \mathrm{kg} / \mathrm{day}$ (maximum daily dose: $15 \mathrm{mg}$ ) in three divided doses, which were tapered over one week.

Periodic (1- to 6-month) follow-up evaluations were performed based on a physical examination, color Doppler flow imaging, CT with 3-dimensional reconstruction, and/or MRI findings during multisession therapy at a congenital vascular malformation clinic. An additional embolization was recommended if symptoms and signs remained or if AVMs remained on follow-up imaging studies.

\section{Evaluation of Therapeutic Outcome and Complications}

A total of 51 ethanol embolization procedures (mean 2.7, range $1-8$ ) were performed in 19 patients. In 14 patients that required two or more procedures, the time interval between the procedures ranged from 1 to 16 months (mean 3.7 months, median 3 months). Clinical follow-up after the last treatment session (mean 22.2 months, range 1-53 months, median 20 months) was performed for all patients and results from imaging followup after the last treatment session (mean 22.1 months, range $2-53$ months, median 21 months) were available for 14 patients.

Two radiologists analyzed by consensus the therapeutic response to ethanol embolization by comparing the degree of AVM devascularization $(100 \%, 76-99 \%, 50-75 \%$, or $<50 \%$ ) between baseline and final angiography. The clinical response (resolved completely, resolved partially, no change, or aggravated) and the presence of complications (major or minor) were evaluated at a congenital vascular malformation clinic by a consensus of two vascular surgeons and one interventional radiologist.

Therapeutic outcome was assessed according to the clinical response and the degree of devascularization at angiography $(10,12)$. Cure was defined as complete resolution of clinical symptoms and signs, with $100 \%$ devascularization of AVMs at angiography. Improvement was defined as complete or partial resolution of clinical symptoms and signs, with 50-99\% AVM devascularization at angiography. No change was defined as partial resolution or no change of clinical symptoms and signs, with $<50 \%$ devascularization at angiography. Aggravation was defined as worsening of clinical symptoms and signs, regardless of the degree of AVM devascularization at angiography. Cure and improvement were considered effective therapeutic outcomes of ethanol embolization of the AVMs.

Major complications included death, permanent adverse sequelae, the requirement for major therapy, and prolonged hospitalization ( $>48$ hours). Minor complications included any non-permanent adverse sequelae, such as a spontaneously healed skin injury.

\section{RESULTS}

The treatment results of ethanol embolization for the 19 patients with peripheral AVMs with a DOV are presented in Table 1. During the 51 sessions of ethanol embolizations, the amount of ethanol used ranged from 2 to $74 \mathrm{~mL}$ in a single session (mean: $34.4 \mathrm{~mL}$, median: $36 \mathrm{~mL}$ ). Flow occlusion techniques to achieve vascular stasis were applied in all patients, but not in all embolization sessions. Flow occlusion techniques were applied in 37 of 51 embolization sessions, including coil and/or core-removed guide wire embolization of the outflow vein in 20 sessions, application of an intravascular occlusion balloon catheter in two sessions, both of the aforementioned methods in three sessions, or application of an external pneumatic cuff in 12 sessions. For 14 embolization sessions, ethanol embolization was performed without an application of flow occlusion techniques as the residual AVM was small or because the application of flow occlusion techniques could induce reflux of ethanol into the adjacent normal arteries. Coil and/or core-removed guide wire embolization of the outflow vein prior to ethanol injection was performed in 13 (68\%) of 19 patients. Coil embolization was performed in seven patients (Fig. 2), core-removed guide wire embolization was performed in two patients, and both coil embolization and core-removed guide wire embolization was performed in four patients. The direct puncture approach was used in 31 of 51 embolization sessions, the transarterial approach was used in 16 sessions, and the transvenous approach was used in eight sessions (multiple approaches in four sessions). Embolization through direct puncture and/or transvenous approaches was performed in 17 of 19 AVMs with a DOV. In the other two patients, ethanol embolization was performed only through a transarterial approach.

Ethanol embolization achieved an effective therapeutic outcome in all 19 patients. Thirteen $(68 \%)$ of the 19 patients were cured, and there was no evidence of recurrence at imaging and clinical follow-up. In the two patients that were cured (patients 8 and 13) who underwent ethanol and core-removed guide wire 


\section{Ethanol Embolization in Peripheral AVM with Dominant Outflow Vein}

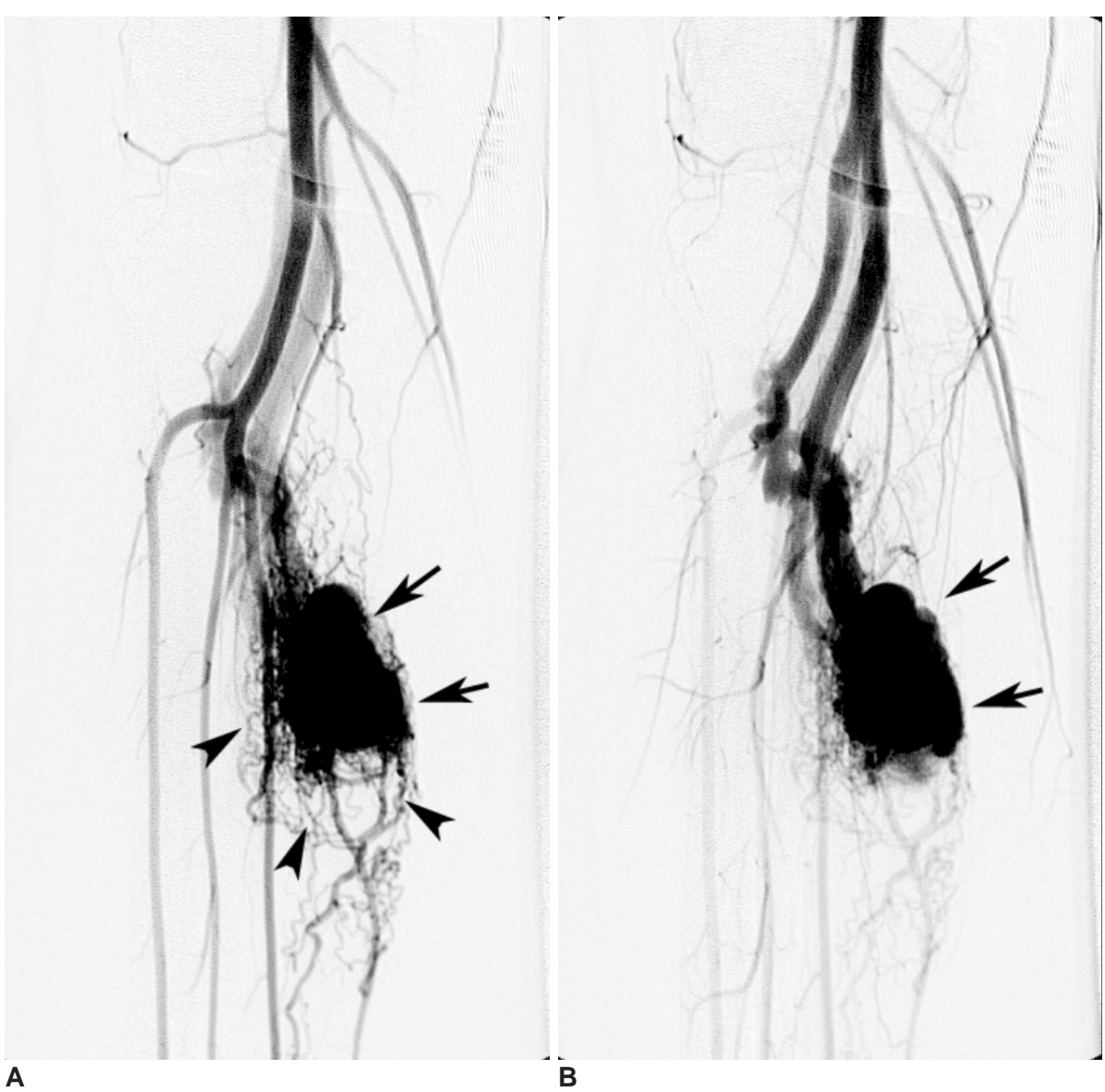

Fig. 2. 23-year-old woman with pain at left calf (patient 5).

A, B. Arterial $(\mathbf{A})$ and venous $(\mathbf{B})$ phases of pretreatment posteroanterior angiogram show multiple feeding arteries (arrowheads) with plexiform appearance and dilated outflow vein (arrows).

C. Multiple coils (arrows) were placed into dilated outflow vein through direct puncture approach, and then $19 \mathrm{~mL}$ of absolute ethanol was injected through needle.

D. Final posteroanterior angiogram shows complete obliteration of arteriovenous malformation. There was no evidence of recurrence at 40-month imaging and clinical follow-up (data not shown).
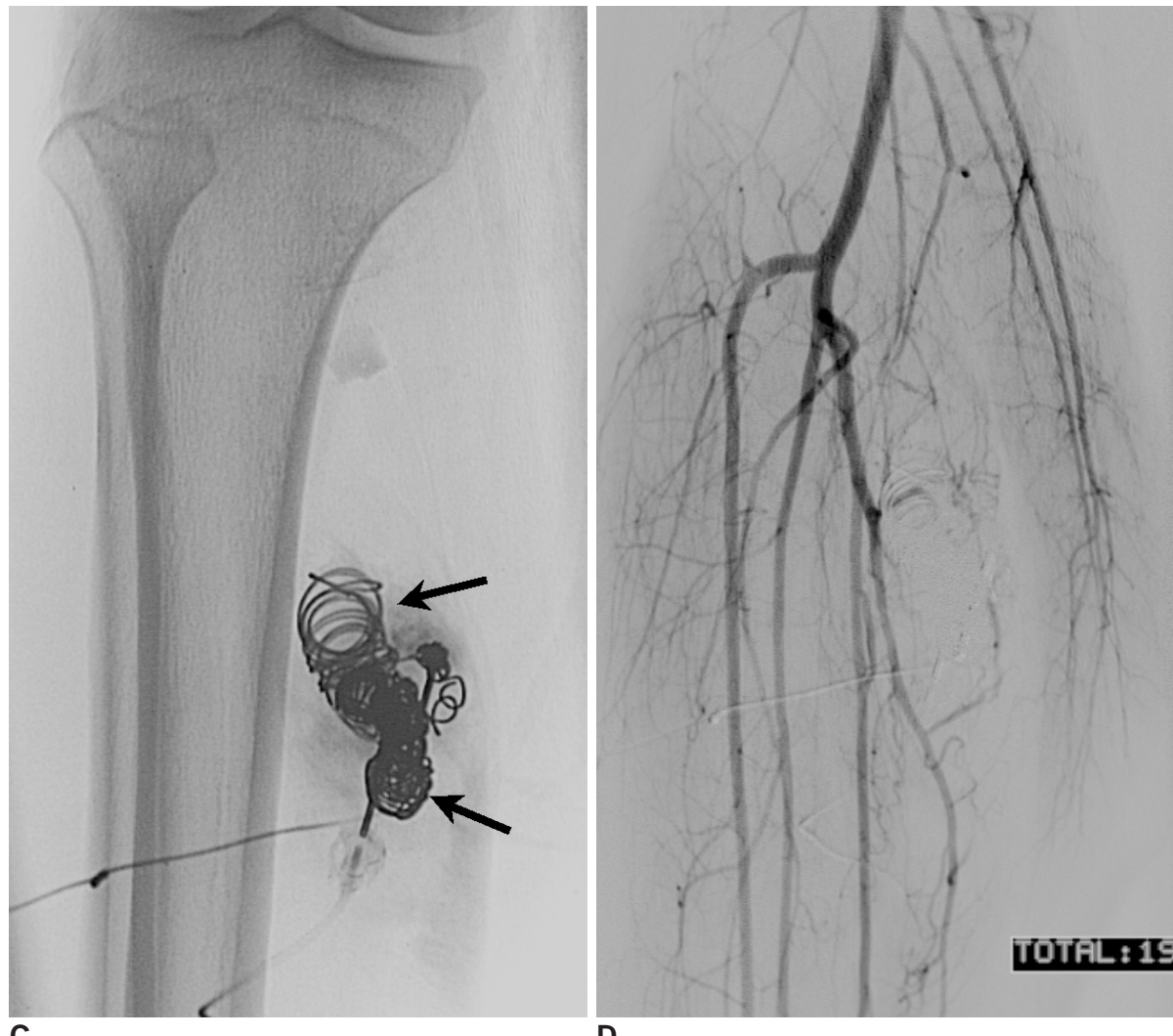
embolization, devascularized AVMs that contained coreremoved guide wires were surgically removed to reduce the mass effect of the guide wires, at one month and two months after completion of embolization, respectively (Fig. 3). Three of the six patients with improvement, in whom clinical symptoms and signs were completely resolved and $\geq 75 \%$ of the AVMs were devascularized, finished treatment as the small residual AVMs were difficult to access or treat safely. The other three patients with improvement, in whom clinical symptoms and signs remained, were waiting for further sessions of ethanol embolization to treat the residual AVMs.

Eight complications (16\%) were encountered in 51 treatment sessions and in four (21\%) of 19 patients. Five complications (three events of a distal embolism and one event of a brain infarct and one event of urinary bladder necrosis) were major and three complications (skin necrosis) were minor. One patient (patient 15) had three events of a distal embolism and two events of skin necrosis during eight embolization sessions. Three events of a distal embolism at the fingers occurred after ethanol embolization alone or in conjunction with coil embolization by the direct puncture approach; two of the embolisms were resolved by continuous infusion of urokinase overnight, but the last embolism required amputation of the fifth finger. Focal necrosis of the urinary bladder occurred after complete embolization of an AVM in the pelvis, which healed in two weeks. The brain infarct was related to the accidental cannulation of the common carotid artery during insertion of the Swan-Ganz catheter. All of the

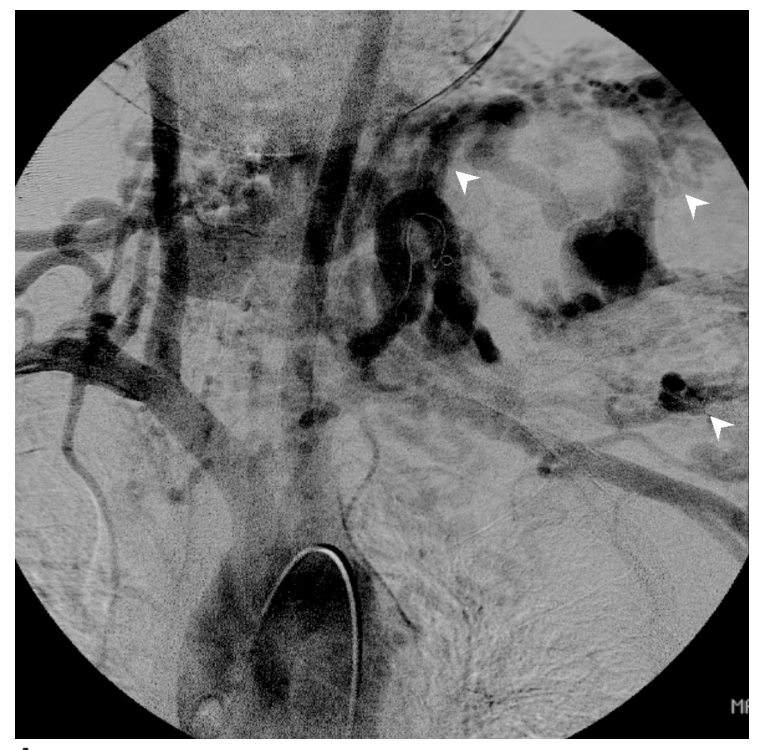

A

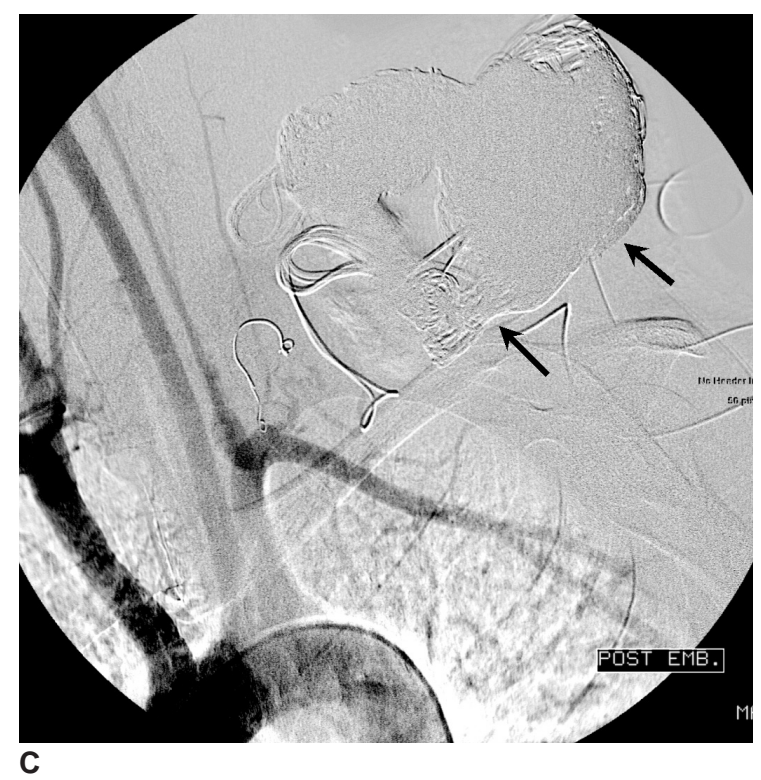

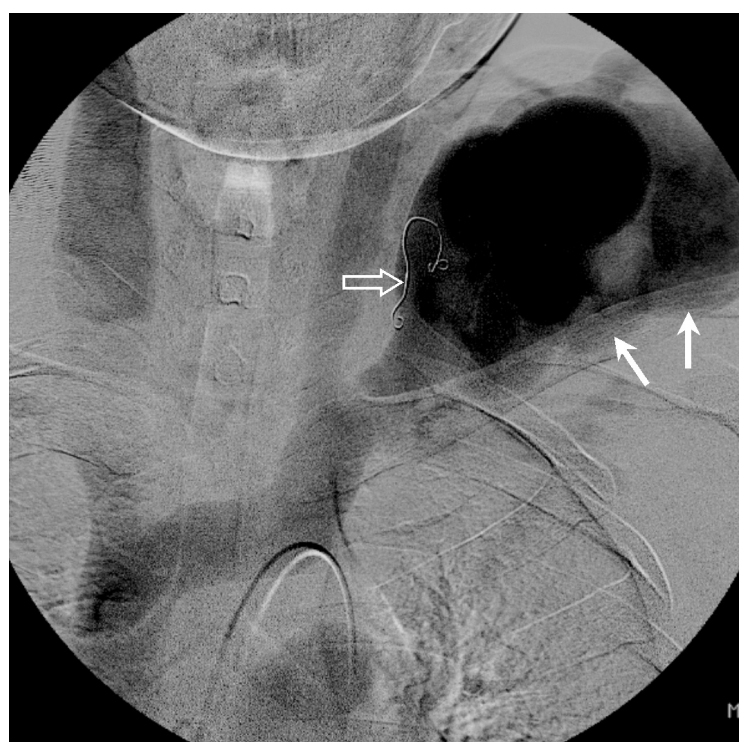

B

Fig. 3. 28-year-old man with painful pulsating mass in left shoulder (patient 8).

A, B. Arterial (A) and venous (B) phases of pretreatment posteroanterior angiogram show multiple feeding arteries (arrowheads) and huge dilated outflow vein (arrows). Note coil (open arrow) that was previously placed into feeding artery at another hospital.

C. Arterial phase of left anterior oblique angiogram shows complete obliteration of arteriovenous malformations after three sessions of embolization with $40 \mathrm{~mL}, 55 \mathrm{~mL}$, and $16 \mathrm{~mL}$ of absolute ethanol, respectively, by using direct puncture of dilated outflow vein. In every session, multiple core-removed guide wires were placed into outflow vein through direct puncture approach prior to ethanol injection. Total of 87 core-removed guide wires (arrows) were placed into outflow vein. Patient complained of discomfort due to mass effect of guide wires after completion of embolization and two months later, patient underwent surgical removal of arteriovenous malformation containing guide wires without any complications. 


\section{Ethanol Embolization in Peripheral AVM with Dominant Outflow Vein}

three skin necroses healed with wound dressing and a skin graft was not required. There was no procedure-related mortality.

\section{DISCUSSION}

Arteriovenous malformations with a DOV are found in about $4 \%$ to $20 \%$ of peripheral AVMs $(9,12,14)$. In the present study, where the incidence of AVMs with a DOV was $17 \%$ (19 of 111 cases), ethanol embolization alone or in conjunction with coil and/or core-removed guide wire embolization achieved effective therapeutic outcomes in all 19 patients. Thirteen patients $(68 \%)$ were cured of the AVMs and six patients achieved improvement during a clinical follow-up period of 1-53 months and an imaging follow-up period of $0-53$ months. In a previous study, Jackson et al. (9) reported that four AVMs with a DOV were effectively treated after ethanol or N-butyl cyanoacrylate embolization and Yee and Yakes (13) reported that 27 patients with AVMs containing a DOV were cured by the use of ethanol alone or in conjunction with coil embolization. These results are better than the outcomes for peripheral AVMs overall $(8,10,11,16,17)$. Accordingly, Cho et al. (12) reported that the therapeutic outcome of AVMs with a DOV was better than that of the other types of AVMs in a study where the investigators analyzed therapeutic results of ethanol embolization in 66 AVM patients according to an angiographic classification. Thus, interventional radiologists performing embolotherapy should realize that peripheral AVMs with a DOV might be effectively treated with a high cure rate.

The nidus of AVMs with a DOV consists of multiple arteriolar components and a dilated venous component, and thus it is clear that approaching the dilated venous component is easier and simpler than superselective positioning to the multiple tortuous arteriolar feeders. Furthermore, based on the present and previous studies (9, 13), an effective therapeutic outcome has mostly been achieved by embolization through the direct puncture and transvenous approaches. Jackson et al. (9) and Yee and Yakes (13) effectively treated AVM patients that had a DOV by embolotherapy through direct puncture and/or transvenous approaches. In the present study, direct puncture and/or transvenous approaches were required for achieving effective embolization in 17 of 19 AVMs with a DOV. Although two AVM patients were treated only with the transarterial approach, transarterial embolization was considered less effective in our initial experience while the transarterial approach was preferentially used to embolize AVMs. Therefore, we believe that the mainstay therapeutic approach for embolizing peripheral AVMs with a DOV should be the direct puncture or transvenous approach.

As the main target of peripheral AVMs with a DOV is the venous component of the nidus, liquid or particulate embolic materials injected through the puncture or transvenous approach may be swept away from the venous side before the materials have managed to achieve occlusion. Even if ethanol, the most powerful sclerosant, is used, the injected ethanol may become diluted with blood and may be swept away from the nidus before it causes nidus occlusion, assuming that the nidus of the AVM type had a large venous component. Thus, flow occlusion techniques are required for achieving effective ethanol embolization of this subset of peripheral AVMs. Jackson et al. (9) used external pneumatic pressure cuffs and intravascular occlusion balloon catheters to achieve vascular stasis of four AVMs. Coil embolization of the DOV prior to ethanol injection was preferred in the present study and previously by Yee and Yakes (13). This preference was a because 1) coil embolization of the DOV can reduce the rate and volume of blood flow within the venous component of the nidus, thereby reducing the amount of ethanol required; 2) the coil(s) can hold a large thrombus that is formed within the large venous component, which can prevent a pulmonary embolism. Additionally, we effectively treated six AVMs with a larger DOV, for which coil embolization was not sufficient and use of an external pneumatic pressure cuff was not possible, by combining the use of ethanol and core-removed guide wire embolization. Based on our results, core-removed guide wire embolization of the outflow vein with a larger size can be one method to make ethanol embolization effective. However, coil or guide wire embolization has some drawbacks. These drawbacks are that coils or guide wires in a very superficial location can erode through the overlying skin and cause ulceration, and in addition, patients can feel some discomfort due to the mass effect (9). Two patients complained of discomfort due to the mass effect of the core-removed guide wires after completion of embolization, and the devascularized AVMs containing the guide wires were surgically removed without any complication one month and two months later, respectively.

Other liquids such as cyanoacrylate and particulate compounds such as polyvinyl alcohol have been used as embolic agents for the treatment of peripheral AVMs (1619). An ideal cyanoacrylate mixture polymerizes in the nidus and partial or total occlusion of the nidus can be achieved. Jackson et al. (9) described two cases of AVMs with a DOV that were treated by using n-butyl cyanoacrylate. However, when this agent is used, it is difficult to control the level of occlusion and there is a risk of a pulmonary embolism from the escape of the agent. It has 


\section{Cho et al.}

also been reported that lesions treated with cyanoacrylate can recanalize $(19,20)$. Particulate agents such as polyvinyl alcohol can induce acute occlusion of AVMs; however, the lesions usually recanalize during follow-up as the nidus is not permanently occluded $(18,21)$. In addition, these agents may be of limited value for the treatment of peripheral AVMs with a DOV as particles are usually used for transarterial embolization.

In the present study, eight (16\%) complications were encountered in 51 treatment sessions and in four $(21 \%)$ of 19 patients. These results are comparable to the complication rates $(10-30 \%)$ of ethanol embolization of peripheral AVMs overall $(3,6)$. Reportedly, the most common complications of ethanol embolization are local tissue injuries, such as skin necrosis and neuropathy (10). We encountered three events of skin necrosis at the treated area and one case of focal urinary bladder necrosis. These local tissue injuries may be associated with non-target embolization or ethanol leakage into the adjacent tissues during embolization. Ethanol injection into the venous component of the nidus when the venous outflow is occluded can cause retrograde filling of the arterial feeding vessels and eventually reflux of ethanol or a thrombus into the arterial side of the circulation (9). Although Jackson et al. (9) and Yee and Yakes (13) previously reported no cases of distal embolism, one patient in our study experienced three events of distal embolism at the fingers. We started overnight urokinase thrombolysis, as it was very difficult to differentiate the cause of the distal embolism on angiography. Two events of distal embolism responded well to thrombolysis, and we presumed that the cause of distal embolism might be reflux of a thrombus and not due to the ethanol. The aforementioned local complications can be minimized by superselective placement of the catheter tip or the needle tip, by strictly performing test injections of contrast medium prior to ethanol injection, and by the meticulous analysis of angiograms during embolization. Although the ethanol washed out from the nidus of an AVM becomes diluted after injection, it can strongly influence the pulmonary artery pressure (3). If pulmonary hypertension that is induced by ethanol injection is not controlled, serious complications may occur, such as a cardiac arrhythmia and cardiopulmonary collapse (10). Thus, monitoring of pulmonary and systemic arterial pressure is essential to minimize the possibility of such potential complications.

This study has some limitations. First, this is a retrospective study with a small number of patients. There is a need for a prospective study involving a significantly large patient population that would permit assessment that is more precise. However, the 19 patients in this study were collected over a 10-year period. The small number of patients may be due to the rarity of peripheral AVMs in general and specifically to the rarity of AVMs with a DOV. Second, the inclusion of the three AVM patients that were waiting for a further session of staged ethanol embolotherapy is another limitation of this study, as the outcome of these patients could change. However, if the patients awaiting further treatment were excluded, this would lead to a selection bias, as only AVMs that responded more easily to treatment would be included in the study.

In conclusion, peripheral AVMs with a DOV can be effectively treated with a high cure rate by the use of ethanol embolization alone or in conjunction with the use of coil and/or core-removed guide wire embolization.

\section{References}

1. Nakano J, Deschryver C. Effects of arteriovenous fistula on systemic and pulmonary circulations. Am J Physiol 1964;207:1319-1324

2. Pritchard DA, Maloney JD, Bernatz PE, Symmonds RE, Stanson AW. Surgical treatment of congenital pelvic arteriovenous malformation. Mayo Clin Proc 1978;53:607-611

3. Yakes WF, Rossi P, Odink H. How I do it. Arteriovenous malformation management. Cardiovasc Intervent Radiol 1996;19:65-71

4. Kaufman SL, Kumar AA, Roland JM, Harrington DP, Barth $\mathrm{KH}$, Haller JA Jr, et al. Transcatheter embolization in the management of congenital arteriovenous malformations. Radiology 1980;137:21-29

5. Widlus DM, Murray RR, White RI Jr, Osterman FA Jr, Schreiber ER, Satre RW, et al. Congenital arteriovenous malformations: tailored embolotherapy. Radiology 1988;169:511-516

6. Yakes WF, Luethke JM, Merland JJ, Rak KM, Slater DD, Hollis HW, et al. Ethanol embolization of arteriovenous fistulas: a primary mode of therapy. J Vasc Interv Radiol 1990;1:89-96

7. Yakes WF, Pevsner P, Reed M, Donohue HJ, Ghaed N. Serial embolizations of an extremity arteriovenous malformation with alcohol via direct percutaneous puncture. AJR Am J Roentgenol 1986;146:1038-1040

8. Gomes AS. Embolization therapy of congenital arteriovenous malformations: use of alternate approaches. Radiology 1994;190:191-198

9. Jackson JE, Mansfield AO, Allison DJ. Treatment of high-flow vascular malformations by venous embolization aided by flow occlusion techniques. Cardiovasc Intervent Radiol 1996;19:323-328

10. Do YS, Yakes WF, Shin SW, Lee BB, Kim DI, Liu WC, et al. Ethanol embolization of arteriovenous malformations: interim results. Radiology 2005;235:674-682

11. Tan KT, Simons ME, Rajan DK, Terbrugge K. Peripheral highflow arteriovenous vascular malformations: a single-center experience. J Vasc Interv Radiol 2004;15:1071-1080

12. Cho SK, Do YS, Shin SW, Kim DI, Kim YW, Park KB, et al. Arteriovenous malformations of the body and extremities: analysis of therapeutic outcomes and approaches according to a modified angiographic classification. J Endovasc Ther 2006;13:527-538

13. Yee DC, Yakes WF. Dominant outflow vein occlusion of high- 


\section{Ethanol Embolization in Peripheral AVM with Dominant Outflow Vein}

flow malformations [abstract]. Presented at the 28th Annual Meeting of the Society of Interventional Radiology, March 2003, Salt Lake City, Utah, USA

14. Houdart E, Gobin YP, Casasco A, Aymard A, Herbreteau D, Merland JJ. A proposed angiographic classification of intracranial arteriovenous fistulae and malformations. Neuroradiology 1993;35:381-385

15. Shin BS, Do YS, Lee BB, Kim DI, Chung IS, Cho HS, et al. Multistage ethanol sclerotherapy of soft-tissue arteriovenous malformations: effect on pulmonary arterial pressure. Radiology 2005;235:1072-1077

16. White RI Jr, Pollak J, Persing J, Henderson KJ, Thomson JG, Burdge CM. Long-term outcome of embolotherapy and surgery for high-flow extremity arteriovenous malformations. J Vasc Interv Radiol 2000;11:1285-1295

17. Osuga K, Hori S, Kitayoshi H, Khankan AA, Okada A, Sugiura $\mathrm{T}$, et al. Embolization of high flow arteriovenous malformations: experience with use of superabsorbent polymer microspheres. $J$ Vasc Interv Radiol 2002;13:1125-1133

18. Palmaz JC, Newton TH, Reuter SR, Bookstein JJ. Particulate intraarterial embolization in pelvic arteriovenous malformations. AJR Am J Roentgenol 1981;137:117-122

19. Schweitzer JS, Chang BS, Madsen P, Vinuela F, Martin NA, Marroquin CE, et al. The pathology of arteriovenous malformations of the brain treated by embolotherapy. II. Results of embolization with multiple agents. Neuroradiology 1993;35:468-474

20. Rao VR, Mandalam KR, Gupta AK, Kumar S, Joseph S. Dissolution of isobutyl 2-cyanoacrylate on long-term follow-up. AJNR Am J Neuroradiol 1989;10:135-141

21. Germano IM, Davis RL, Wilson CB, Hieshima GB. Histopathological follow-up study of 66 cerebral arteriovenous malformations after therapeutic embolization with polyvinyl alcohol. J Neurosurg 1992;76:607-614 